\title{
Metaphoric optical computing for fluid dynamics
}

\section{Mankei Tsang, Demetri Psaltis}

Mankei Tsang, Demetri Psaltis, "Metaphoric optical computing for fluid dynamics," Proc. SPIE 5735, Advanced Optical and Quantum Memories and Computing II, (4 April 2005); doi: 10.1117/12.601656

Event: Integrated Optoelectronic Devices 2005, 2005, San Jose, California, United States 
Invited Paper

\title{
Metaphoric Optical Computing for Fluid Dynamics
}

\author{
Mankei Tsang and Demetri Psaltis \\ Department of Electrical Engineering, California Institute of Technology, Pasadena, CA 91125
}

\begin{abstract}
We show that an optical pulse inherently computes three-dimensional classical fluid dynamics. Taking optical diffraction, dispersion and nonlinearity into account, one can define the metaphoric fluid density, velocity and vorticity in the optical pulse. We propose the use of the group-velocity-delayed time to represent the third dimension of the fluid, and the "splitstep" method to combine optical devices as a configurable system that simulates fluid flow. Optical systems, with the inherent speed, parallelism and configurability, may one day be utilized to assist the study of fluid dynamics.
\end{abstract}

\section{INTRODUCTION}

It is well known that the nonlinear Schrodinger equation (NLSE), which describes optical propagation, Bose-Einstein condensates and superfluids among many other physical phenomena, can be transformed to hydrodynamical equations. ${ }^{1}$ The hydrodynamical formulation is studied mainly in the field of superfluidity, ${ }^{2}$ while in the field of optics, the fluid description has been used, for example, in the study of laser dynamics, ${ }^{3-5}$ two-dimensional optical flow in a cavity 6,7 and optical vortex dynamics. $^{8-10}$ In this perspective, optics seems to have at least some correspondence to fluid dynamics. A field essential to aeronautics and weather prediction, computational fluid dynamics nonetheless experiences tremendous difficulties due to the overarching computing power and data storage required. While fluid experiments such as wind tunnels can be used to study flow problems, they are not very configurable and it is difficult to specify complex initial conditions. Meanwhile it has been proposed that NLSE can be used to study inviscid fluid dynamics, ${ }^{11}$ the dynamics of NLSE exhibits classical turbulence behavior ${ }^{12}$ and light propagation can be used to study properties of superfluids. ${ }^{13}$ This makes one wonder if a much larger class of problems in fluid dynamics can be studied with the help of optical systems, which inherently display speed, parallelism and configurability.

Building on previous attempts that relate light to two-dimensional fluids or superfluids, our ultimate target is to use optics to accurately simulate three-dimensional ordinary fluids in high-Reynolds-number flow. The optical intensity and the local wave vector can represent the fluid density and velocity respectively, the optical pulse shape can be used to represent the third dimension of the fluid if group-velocity dispersion is negative, and mirrors with small structures can be introduced to implement an effective "no-slip" boundary condition. This correspondence to classical fluids may one day be used as an "optical wind tunnel" for the metaphoric computing of fluid dynamics.

While in theory nonlinear optical propagation can accurately model fluid flow, an ideal nonlinear medium in which the correspondence is exact may not be easily fabricated. We hereby introduce the "split-step" method, in which propagation effects can be applied separately with different optical devices to an optical pulse, so that the final outcome will approximate the true solution as if we had the ideal medium.

\section{OPTICAL ANALOGUES OF FLUID DENSITY AND VELOCITY}

To begin, let us reproduce the Madelung transformation of NLSE to fluid equations, ${ }^{1}$ with the inclusion of the pulse shape as the third dimension. The nonlinear Schrodinger equation in optics is given by

$$
\frac{\partial A}{\partial z}=\frac{j}{2 k_{0}} \nabla_{\perp}^{2} A-\frac{j \beta_{2}}{2} \frac{\partial^{2} A}{\partial t^{2}}+j k_{0} \Delta n\left(\mathbf{r}_{\perp}, t,|A|^{2}\right) A
$$

where $A=|A(z, x, y, t)| \exp [j \psi(z, x, y, t)]$ is the complex envelope, $k_{0}=2 \pi / \lambda_{0}$ is the carrier wave number, $\nabla_{\perp}^{2}$ is the transverse Laplacian, $\beta_{2}$ is the group-velocity dispersion coefficient, $t$ is the group-velocity-delayed time, and $\Delta n$ is the refractive index change that can be due to the spatial refractive index profile of the medium, or induced by nonlinear self- or cross-phase modulation. For the Kerr nonlinearity, $\left.\Delta n\right|_{\text {Kerr }}=n_{2}|A|^{2}$. Dispersion can be considered as the third dimension of diffraction if the group-velocity dispersion is anomalous, or $\beta_{2}<0$. The corresponding spatial coordinate can then be defined as $\tau \equiv t / \sqrt{-\beta_{2} k_{0}}$. 
The Madelung transformation is given by

$$
\begin{aligned}
i & \equiv|A|^{2} \\
\kappa & \equiv \nabla^{\prime} \psi=\left(\hat{\mathbf{r}}_{\perp} \nabla_{\perp}+\hat{\boldsymbol{\tau}} \frac{\partial}{\partial \tau}\right) \psi
\end{aligned}
$$

where $i$ is the intensity and $\kappa$ is the local wave vector. Two equations are thus produced,

$$
\begin{aligned}
\frac{\partial i}{\partial z}+\frac{1}{k_{0}} \nabla^{\prime} \cdot(i \boldsymbol{\kappa}) & =0 \\
\frac{\partial \boldsymbol{\kappa}}{\partial z}+\frac{1}{k_{0}} \nabla^{\prime}\left(\frac{1}{2} \kappa^{2}\right) & =\nabla^{\prime}\left(k_{0} \Delta n\right)+\frac{1}{k_{0}} \nabla^{\prime}\left(\frac{1}{2 \sqrt{i}} \nabla^{\prime 2} \sqrt{i}\right) .
\end{aligned}
$$

Eq. (4) is identical to the continuity equation in hydrodynamics, with propagation displacement $z$ acting as time, optical intensity $I$ as fluid density and $\kappa$ as velocity. The (negative) instantaneous frequency represents the third component of velocity. Eq. (5) resembles the Euler equation of inviscid motion for irrotational flow $(\nabla \times \mathbf{v}=0)$, with $-\Delta n$ acting as pressure. The nonlinearity should be defocusing $\left(n_{2}<0\right)$ so that the pressure term provides the correct fluid behavior. The last term of Eq. (5) is called the "quantum pressure," which is not present in classical fluid dynamics.

If the characteristic intensity of the optical system is $I$, the characteristic length is $W$ and the characteristic local wave number is $K$, Eqs. (4) and (5) can be renormalized according to the following parameters,

$$
\begin{aligned}
\zeta & \equiv \frac{K}{W k_{0}} z, \\
\nabla & \equiv W \nabla^{\prime}, \\
\rho & \equiv \frac{i}{I}, \\
\mathbf{v} & \equiv \frac{\kappa}{K}, \\
a & \equiv \frac{1}{k_{0} \sqrt{-n_{2} I}}, \\
\mathcal{M} & \equiv K a, \\
\mathcal{H} & \equiv \frac{a}{W},
\end{aligned}
$$

where $a$ is called the "healing" length, which characterizes the length over which the intensity bends at a boundary. $\mathcal{M}$ is the Mach number in the fluid picture, and $\mathcal{H}$ is the normalized healing length. The normalized equations of motion are

$$
\begin{aligned}
\frac{\partial \rho}{\partial \zeta}+\nabla \cdot(\rho \mathbf{v}) & =0 \\
\frac{\partial \mathbf{v}}{\partial \zeta}+\nabla\left(\frac{1}{2} v^{2}\right) & =-\frac{1}{\mathcal{M}^{2}} \nabla\left(\rho-\frac{\mathcal{H}^{2}}{2 \sqrt{\rho}} \nabla^{2} \sqrt{\rho}\right) .
\end{aligned}
$$

The "quantum pressure" term gives rise to Bogoliubov dispersion in a superfluid, ${ }^{6}$ but should be made negligible compared to the Kerr pressure term if ordinary fluid behavior is desired, so

$$
\begin{gathered}
\mathcal{H}<<1, \\
W>>a .
\end{gathered}
$$

The Euler equation of irrotational inviscid fluid motion is then obtained,

$$
\frac{\partial \mathbf{v}}{\partial \zeta}+\nabla\left(\frac{1}{2} v^{2}\right)=-\frac{1}{\mathcal{M}^{2}} \nabla \rho
$$


A compressible fluid may demand a different pressure dependence on density. For instance, the pressure term of an isothermal ideal gas is $\propto(1 / \rho) \nabla \rho=\nabla \ln \rho$. Hence for a good correspondence with fluid dynamics, we shall assume a small compressibility, or in terms of the Mach number $\mathcal{M}$,

$$
\mathcal{M}=K a<<1,
$$

so that Kerr nonlinearity is a good first-order approximation. A small compressibility also helps to ensure a small "quantum pressure" term, so that the requirement in Eq. (16) can be somewhat relaxed.

\section{VORTICITY}

While the Madelung transformation relates nonlinear optical propagation to irrotational flow, most interesting fluid problems concern the concept of vorticity, which is responsible for turbulence. It is defined as

$$
\boldsymbol{\omega} \equiv \nabla \times \mathbf{v} .
$$

Optical vorticity does exist, but in the quantized form of optical vortices. For a three-dimensional optical pulse that includes the dispersion effect vortices exist in the form of vortex lines, just like in superfluids. For a direct correspondence between optics and classical hydrodynamics it is therefore essential to relate the quantized vortex lines to the distributed vorticity.

The magnitude of vorticity can be estimated by the closed path integral of $\mathbf{v}$,

$$
\begin{aligned}
\boldsymbol{\omega} \cdot \hat{\mathbf{n}} & =\lim _{\sigma \rightarrow 0} \frac{1}{\sigma} \oint \mathbf{v} \cdot d \mathbf{l} \\
& =\lim _{\sigma \rightarrow 0} \frac{1}{\sigma} \oint \nabla \psi \cdot d \mathbf{l},
\end{aligned}
$$

where $\hat{\mathbf{n}}$ is the unit vector in the direction of $\boldsymbol{\omega}, \sigma$ is the area near the position of the vorticity to be calculated, and the path integral is around the area of interest. The path integral is the accumulation of phase when one goes around a closed loop, which can be multiples of $2 \pi$ and is called the circulation. A non-zero circulation can only exist near an optical vortex, and as such if the path integral is not around a vortex the vorticity is zero, otherwise it is infinite. This can be written in a mathematical form as

$$
\begin{aligned}
\boldsymbol{\omega}(\mathbf{r}) & =\sum_{j} \boldsymbol{\omega}_{j}(\mathbf{r}) \\
& =\sum_{j} \Gamma_{j} \int d \mathbf{r}_{j} \delta\left(\mathbf{r}-\mathbf{r}_{j}\right),
\end{aligned}
$$

where $\Gamma_{j}$ is the circulation around a vortex denoted by $j, \delta\left(\mathbf{r}-\mathbf{r}_{j}\right)$ is a three-dimensional Dirac delta function, $\mathbf{r}=$ $(x \hat{\mathbf{x}}+y \hat{\mathbf{y}}+\tau \hat{\boldsymbol{\tau}}) / W$ is the normalized position vector, $\mathbf{r}_{j}=\left[x_{j}(\xi, \zeta) \hat{\mathbf{x}}+y_{j}(\xi, \zeta) \hat{\mathbf{y}}+\tau_{j}(\xi, \zeta) \hat{\boldsymbol{\tau}}\right] / W$ denotes the position of a vortex line (parametrized by $\xi$ ) and the integral is performed along a vortex line.

The intensity at the center of the vortex is required to be zero, and the size of the intensity null is on the order of the healing length $a$ as given by Eq. (10). Compressible effects due to the intensity null are minimized on the macroscopic scale if the approximation given by Eq. (16) is satisfied.

We can now patch up the equation of motion, Eq. (17), to include the effect of vortices, where the Madelung transformation is undefined because $\rho$ is zero, ${ }^{2,15}$

$$
\frac{\partial \mathbf{v}}{\partial \zeta}+\nabla\left(\frac{1}{2} v^{2}\right)+\boldsymbol{\omega} \times \mathbf{v}=-\frac{1}{\mathcal{M}^{2}} \nabla \rho
$$

The inclusion of the term $\boldsymbol{\omega} \times \mathbf{v}$ can be explained by the phenomenon of phase slip. ${ }^{2}$

Vortex lines with the lowest circulation possible are the most energetically stable and we can therefore safely assume that $\Gamma_{j}=2 \pi$ when vortex lines are naturally generated, for example, by a boundary. Furthermore, the vorticity can be 
regarded as continuous if the total absolute circulation of a given problem, $\sum_{j}\left|\Gamma_{j}\right| \sim K W$, is shared by a large number of vortices. We can therefore approximate continuous vorticity with quantized vortices if

$$
K W>>2 \pi .
$$

In other words, when the requirement of Eq. (25) is satisfied, the quantization of vortices plays a much smaller role and the dynamics should approach the classical behavior. The use of discrete vortices to model continuous vorticity is a wellestablished numerical method in fluid dynamics. ${ }^{16}$ Simulations ${ }^{11}$ also show that the use of NLSE is a valid alternative to the Euler and Navier-Stokes equations for the study of free shear flow, and the NLSE dynamics of a large number of vortices can exhibit classical turbulence behavior. ${ }^{12}$

The formalism above shows that inviscid fluid dynamics can be studied by nonlinear optical propagation. 2D initial conditions can be specified using, for instance, spatial light modulators or holography. For 3D initial conditions, complete spatiotemporal amplitude and phase specification is needed, although current technology is not yet able to do so efficiently. Nonetheless one can certainly envision the use of short-pulse wave mixing, cross-phase modulation, or even spatial light modulators embedded in a slow light medium to accomplish the task. 3D amplitude and phase of the output optical beam can be measured by short-pulse holography.

\section{BOUNDARY CONDITIONS}

The refractive index acts as a (negative) potential function in the NLSE. A low refractive index region compared to the bulk thus corresponds to a high potential area. In the limit of infinite potential, the density $\rho$ is restricted to be zero, and equivalently the velocity component normal to the boundary is set to zero, while there is no restriction on the tangential component. This is called the "free-slip" boundary condition in fluid dynamics.

To enforce a 3D boundary condition, the refractive index profile must be in 3D ( $x, y$ and $t)$ as well, which means that the boundary must co-propagate with the pulse. One can therefore only use another light pulse, with a different polarization or frequency, to induce a 3D refractive index profile via cross-phase modulation. The "boundary" pulse should not change significantly with respect to $z$, so one should periodically compensate for its diffraction and dispersion, with, for instance, optical phase conjugation.

While one can enforce the "free-slip" boundary condition in optics, the most commonly used boundary condition in fluid dynamics is the "no-slip" boundary condition, which mandates a zero total velocity at the interface and has no obvious equivalence in optics. This issue is tackled in the next section.

\section{VORTEX NUCLEATION}

In an ordinary fluid, the creation of vortices is mainly due to an obstacle in the flow, the boundary of which in most cases is "no-slip". The vorticity naturally generated at such a boundary is diffused into the main flow by viscosity.

In optics one can only have a "free-slip" boundary as enforced by the refractive index. In a completely incompressible fluid, a "free-slip" boundary only results in a potential flow and does not generate vorticity.

Since a "no-slip" boundary, and viscosity for that matter, do not naturally exist in optics, we are forced to find alternative methods to mimic the ordinary fluid behavior of vorticity nucleation and diffusion. One method to generate vortices at a "free-slip" boundary is by compressibility, when velocity anywhere in the flow exceeds the speed of sound. ${ }^{14}$ This seems to violate our assumption of incompressibility, but all we require is a high velocity near the boundary in order to generate vortices, while the velocity some distance away from the boundary can remain well below the speed of sound so that compressible effects are not large overall. Indeed, for a potential flow around a cylinder, the critical Mach number $\mathcal{M}_{c}$ at which vortices start to form around the cylinder is only about $0.4 .{ }^{14}$

To minimize compressible effects, one would like to lower the critical Mach number. This can be done by introducing sharp corrugations on the wall of a boundary, ${ }^{14}$ so that the potential flow speed near each corrugation is multiplied by its aspect ratio, given by the height of each corrugation $h$ divided by its width $w$. In other words, the critical Mach number is proportional to $w / h$. We can then achieve vortex emission in the small compressibility regime if

$$
\mathcal{M}_{c} \sim \frac{w}{h}<<\mathcal{M}<<1 .
$$


For a sharp corrugation, the potential flow speed quickly reduces to the macroscopic value at a distance $h$ away from the boundary, so as long as $h<<W$ the overall dynamics can still be regarded as incompressible.

What is the equivalent Reynolds number in the optical flow then? Reference ${ }^{12}$ compares the average distance among the vortices to the Taylor microscale, and concludes that the Reynolds number should be given by $\mathcal{R} \sim K W$, according to our normalization. However, the average distance as calculated in Ref. ${ }^{12}$ is only due to the quantized nature of the vortices, while the Taylor microscale is the scale at which viscous effect becomes noticeable. This comparison is thus between two length scales with different physical origins. A more physically sound estimate can be made by observing that the only dissipation mechanism available in a pure superflow is acoustic emission, which only becomes significant when the distance between two vortices is as close as $a$. One should then compare the Taylor microscale to $a$ instead, or

$$
\begin{aligned}
a & \sim \frac{W}{\sqrt{\mathcal{R}}}, \\
\mathcal{R} & \sim \frac{1}{\mathcal{H}^{2}} .
\end{aligned}
$$

\section{THE SPLIT-STEP METHOD}

While an optical system shows promise for computing fluid dynamics, it also poses serious technical challenges. Ideally one would like to have a configurable nonlinear material with low loss, anomalous group-velocity dispersion, high defocusing nonlinearity, tailored spatial and temporal coherent diffusion properties and three-dimensional co-propagating boundaries. One may only be able to find separate materials or optical devices, each of which performs only some of the functions. Moreover, parasitic effects such as loss and high-order dispersion can be detrimental to the accuracy. To combine different devices and periodically compensate for parasitic effects, one may use the so-called "split-step" method. Consider the general NLSE,

$$
\frac{\partial A}{\partial \zeta}=\sum_{n=1}^{N} \hat{H}_{n} A
$$

where propagation effects and boundary conditions are expressed in terms of operators $\hat{H}_{n}$. The formal solution is

$$
A(\zeta+\Delta \zeta)=\exp \left(\int_{\zeta}^{\zeta+\Delta \zeta} \sum_{n=1}^{N} \hat{H}_{n} d \zeta^{\prime}\right) A(\zeta)
$$

But if $\Delta \zeta$ is much smaller than $1 / H$ where $H$ is the magnitude of the operators, by virtue of the Baker-Hausdorff formula we have

$$
A(\zeta+\Delta \zeta)=\prod_{n=1}^{N} \exp \left(\hat{H}_{n} \Delta \zeta\right) A(\zeta)+O\left(H^{2} \Delta \zeta^{2}\right)
$$

Each of the propagation effects can thus be applied separately to an optical pulse, with a quadratic error term. A symmetrized version of the split-step method can further reduce the error order,

$$
A(\zeta+\Delta \zeta)=\prod_{m=N}^{1} \exp \left(\hat{H}_{m} \frac{\Delta \zeta}{2}\right) \prod_{n=1}^{N} \exp \left(\hat{H}_{n} \frac{\Delta \zeta^{\prime}}{2}\right) A(\zeta)+O\left(H^{3} \Delta \zeta^{3}\right) .
$$

One can then form a unit cell of a "meta-material" by combining a slice of defocusing material, a slice of anomalously dispersive material, a nonlinear region to apply the three-dimensional boundary conditions periodically by short pulses, and a gain medium to compensate for loss. The optical pulse can loop through the unit cell multiple times, so that the outcome will approximate the true solution as if we had an ideal medium.

The split-step method has the additional advantages that each subsystem can be tunable and easily substituted with another material or device, and the pulse evolution can be monitored more easily. The magnitude of each effect can be tuned by simply changing the propagation length in each device, In exchange of configurability we have sacrificed some accuracy due to discretization errors and instability. The computation speed may also be reduced by a large but constant fraction, as the pulse may spend most of its time on simply propagating from one device to the next and not performing the core computation by nonlinear propagation. The "split-step" method, however, does not detract from the inherent parallelism in the computation, as the transverse dimensions are not discretized. 


\section{SCALING LAWS}

The first and foremost requirement for our optical system is to ensure an accurate correspondence with classical fluid dynamics. One can obtain the same dynamics with arbitrary scales as long as the Reynolds number $\mathcal{R}$ and the Mach number $\mathcal{M}$ are the same. In terms of fluid characteristic size $L$, velocity $U$, kinematic viscosity $\nu$ and sound speed $c_{s}, \mathcal{R}$ and $\mathcal{M}$ are defined as

$$
\begin{aligned}
\mathcal{R} & \equiv \frac{U L}{\nu}, \\
\mathcal{M} & \equiv \frac{U}{c_{s}} .
\end{aligned}
$$

In terms of optical parameters,

$$
\begin{aligned}
\mathcal{R} & =\frac{U L}{\nu}=\left(\frac{W}{a}\right)^{2}, \\
\mathcal{M} & =\frac{U}{c_{s}}=K a .
\end{aligned}
$$

From the earlier discussions we see that optics can accurately simulate fluids if the Reynolds number is high $(\mathcal{R}>>1)$ and the Mach number is relatively low $(\mathcal{M}<<1)$.

Quantitatively, to ensure accuracy, all the assumptions in the above discussions should be satisfied and can be summarized as

$$
W>>\frac{2 \pi}{K}>>2 \pi a>>\lambda_{0}
$$

A high optical intensity $\rho$ is required to maintain small compressibility. In terms of the characteristic power $P$ and intensity $I$,

$$
P \sim I W^{2}>>\frac{K^{2} W^{2}}{k_{0}^{2} n_{2}} \sim \frac{\mathcal{M}^{2} \mathcal{R}}{k_{0}^{2} n_{2}} .
$$

Hence the total power is required to be much higher than the critical power, defined as $1 /\left(k_{0}^{2} n_{2}\right)$.

To estimate the ultimate performance of an optical system for fluid dynamics computation, we assume that an ideal optical medium can be fabricated and all technical problems can be solved without the need of the "split-step" method. The computation speed is then only limited by how fast an optical pulse can diffract and disperse. The time taken, $t_{o}$, by an optical pulse to propagate and compute the dynamics of a slightly compressible fluid flowing for a duration of $t_{f}$, with the same $\mathcal{R}$ and $\mathcal{M}$, is the propagation distance divided by the group velocity $v_{g}$, provided that the pulse width is not longer than the latency. $t_{o}$ is then given by

$$
\begin{aligned}
t_{o} & \sim \frac{U}{v_{g}} \frac{k_{0}}{K} \frac{W}{L} t_{f} \sim \frac{\mathcal{M}}{\sqrt{\mathcal{R}}} \frac{c_{s}^{2}}{k_{0} \Delta n \nu v_{g}} t_{f} \equiv \frac{1}{S} t_{f} . \\
S & =\frac{\sqrt{\mathcal{R}}}{\mathcal{M}} \frac{k_{0} \Delta n \nu v_{g}}{c_{s}^{2}}
\end{aligned}
$$

The factor $S$ determines the computation speed of an optical system relative to a fluid. For typical values, $\lambda_{0} \sim 1 \mu \mathrm{m}$, $\Delta n \sim 10^{-4}, v_{g} \sim 10^{8} \mathrm{~ms}^{-1}, c_{s} \sim 10^{3} \mathrm{~ms}^{-1}, \nu \sim 10^{-5} \mathrm{~m}^{2} \mathrm{~s}^{-1}, S$ is on the order of $\sqrt{\mathcal{R}} / \mathcal{M}>>1$, which means that optics inherently computes fluid dynamics faster than even the fluid itself.

For an incompressible flow, one can study a fluid problem by experimenting with a smaller model, which evolves faster, while keeping the Reynolds number constant, such as in a wind tunnel. It is, however, impractical to make the model too small because the flow velocity of a pressure-driven flow is limited by the size. Similarly, the size of an optical system can 
be reduced to achieve faster computation of incompressible fluid dynamics, as long as the Reynolds number is the same while the Mach number is small enough. Provided that all other requirements are satisfied, $t_{o}$ in this regime is given by

$$
\begin{aligned}
t_{o} & \sim \frac{W^{2}}{L^{2}} \frac{\sqrt{\mathcal{R}}}{\mathcal{M}} \frac{k_{0} \nu}{v_{g}} t_{f} \equiv \frac{W^{2}}{L^{2}} \frac{1}{S_{I}} t_{f} . \\
S_{I} & =\frac{\mathcal{M}}{\sqrt{\mathcal{R}}} \frac{v_{g}}{k_{0} \nu} .
\end{aligned}
$$

The factor $S_{I}$ determines the computation speed of the optical system relative to an incompressible fluid if the two systems have the same size. Using the parameters above, $S_{I} \sim 10^{6} \mathcal{M} / \sqrt{\mathcal{R}}$. Say $\mathcal{R} \sim 10^{6}, \mathcal{M} \sim 0.1, S_{I} \sim 100$, so the optical system is 100 times faster than the fluid system when the two systems have the same size, while further speed advantage can be gained by reducing the optical system size.

\section{CONCLUSION}

We have established a comprehensive formulation of "optohydrodynamics," in which an optical pulse can exihibit classical fluid properties and physical boundary conditions can be optically implemented. While this formalism is by no means complete because of the restriction of small compressibility and the lack of a fluid energy equation, our theory still obeys the fluid mass and momentum conservation equations, which should faithfully describe a large class of interesting problems including turbulence. Optical effects not considered here, such as incoherence, polarization, non-Kerr-type nonlinear effects and wave mixing, may also be used to further generalize our formulation. For example, incoherence may be related to the temperature of a fluid, ${ }^{7}$ optical vortex solitons, which should exhibit fluidic properties, have been observed in photorefractive ${ }^{19}$ and photovoltaic media ${ }^{20}$ and with partially coherent light. ${ }^{21}$ Optical vortex streets in walking secondharmonic generation have been observed and compared to von Karman vortex streets. ${ }^{22}$

There are enormous technical challenges ahead if actual systems are to be built for the purpose of fluid simulations especially for three-dimensional problems, but the speed, parallelism and configurability of optics promise vast advantages over numerical computation and fluid experiments.

\section{ACKNOWLEDGMENTS}

We thank Dr. Ravi Athale of DARPA and Prof. Michael Cross of Caltech for helpful discussions. Financial support from DARPA is gratefully acknowledged.

\section{REFERENCES}

1. E. Madelung, “Quantetheorie in hydrodynamischer form,” Z. Phys. 40322 (1927).

2. R. J. Donnelly, Quantized Vortices in Helium II (Cambridge University Press, New York, 1991).

3. M. Brambilla, L. A. Lugiato, V. Penna, F. Prati, C. Tamm and C. O. Weiss, Phys. Rev. A 43, 5114 (1991).

4. K. Staliunas, Phys. Rev. A 48, 1573 (1993).

5. M. Vaupel, K. Staliunas and C. O. Weiss, Phys. Rev. A 54, 880 (1996).

6. R. Y. Chiao and J. Boyce, Phys. Rev. A 60, 4114 (1999).

7. E. L. Bolda, R. Y. Chiao and W. H. Zurek, Phys. Rev. Lett. 86, 416 (2001).

8. G. A. Swartzlander, Jr. and C. T. law, Phys. Rev. Lett. 69, 2503 (1992).

9. D. Rozas, C. T. Law and G. A. Swartzlander, Jr., J. Opt. Soc. Am. B 14, 3054 (1997).

10. D. Rozas, Z. S. Sacks and G. A. Swartzlander, Jr., Phys. Rev. Lett. 79, 3399 (1997).

11. C. Nore, M. E. Brachet and S. Fauve, Physica D 65, 154 (1993), C. Nore, M. Abid and M. Brachet, Mecanique des fluids 319, 733 (1994).

12. M. Abid, C. Huepe, S. Metens, C. Nore, C. T. Pham, L. S. Tuckerman, M. E. Brachet, Fluid Dynamics Research 33, 509 (2003), and references therein.

13. Y. Pomeau and S. Rica, C.R. Acad. Sci. Paris 317, 1287 (1993).

14. T. Frisch, Y. Pomeau and S. Rica, Phys. Rev. Lett. 69, 1644 (1992).

15. E. B. Sonin, Rev. Mod. Phys. 59, 87 (1987). 
16. A. Leonard, J. Comp. Phys. 37, 289 (1980).

17. S. Granick, Y. Zhu, H. Lee, Nature Materials 2, 221 (2003).

18. K. M. Jansons, Phys. Fluids 31, 15 (1988).

19. G. Duree, M. Morin, G. Salamo, M. Segev, B. Crosignani, P. Di Porto, E. Sharp, A. Yariv, Phys. Rev. Lett. 74, 1978 (1995).

20. Z. Chen, M. Segev, D. W. Wilson, R. E. Muller, P. D. Maker, Phys. Rev. Lett. 78, 2948 (1997).

21. Z. Chen, M. Mitchell, M. Segev, T. H. Coskun, D. N. Christodoulides, Science 280, 889 (1998).

22. G. Molina-Terriza, D. V. Petrov, J. Recolons, L. Torner, Opt. Lett. 27, 625 (2002). 